\section{IMPACT OF ULTRA-FAST 'FLASH' RADIOTHERAPY ON SINGLE CELL IMMUNOGENOMICS IN DIFFUSE INTRINSIC PONTINE GLIOMA (DIPG)}

'Oscar Padilla*, ${ }^{1}$ Hanna Minns, ${ }^{1}$ Hong-Jian Wei, ${ }^{1}$ Andrea Webster-Carrion, ${ }^{1}$ Masih Tazhibi, 'Nicholas McQuillan, 'Xu Zhang, 'Zhiguo Zhang, 'Raul Rabadan, 'Peter Canoll, ${ }^{1}$ Luca Szalontay, ${ }^{1} J o v a n a$ Pavisic, 'Guy Garty, ${ }^{1}$ Stergios Zacharoulis, ${ }^{2}$ Claire Vanpouille-Box, ${ }^{1}$ Vilas Menon, ${ }^{1}$ Marta Olah, ${ }^{1}$ Cheng-Chia Wu, ${ }^{1}$ Robyn Gartrell. ${ }^{1}$ Columbia University Medical Center, New York, NY, USA; '2Weill Cornell Medicine, New York, NY, USA

Background Diffuse intrinsic pontine gliomas (DIPG's) are immunologically inert tumors with a median survival of 9-15 months. Radiation therapy (RT) is the mainstay treatment for DIPG but is associated with immunodepletion of the tumor microenvironment (TME) at high dose ranges. FLASH, or ultra-fast dose rate RT, represents a novel ablative technique that may spare TME immune responses while decreasing tumor burden. Here, we present single-cell immune profiling of DIPG tumors treated with FLASH, conventional dose rate RT (CONV) or no RT (SHAM).

Methods Murine H3.3K27M mutant DIPG cells were stereotactically injected and tumor induction confirmed by magnetic resonance imaging (MRI) 15 days later. DIPG-bearing mice were randomly assigned to one of three treatment groups ( $n=4 /$ group), FLASH, CONV or SHAM. A fourth group with no tumor (NML) was included as a negative biological control. A modified linear accelerator was used to deliver $15 \mathrm{~Gy}$ of electron RT to the brainstem at dose rates of $90 \mathrm{~Gy} / \mathrm{second}$ and $2 \mathrm{~Gy} /$ minute, for the FLASH and CONV groups, respectively. Four days post-RT, mice brainstems were harvested, homogenized, stained for CD45 and tagged with a hashtag antibody specific to each group. CD45+ immune cells were isolated and sequenced using the $10 \mathrm{X}$ Genomics chromium single-cell 3' platform. After processing and alignment of the reads using CellRanger with default parameters, the data was quality checked and filtered before hashtag demultiplexing, unsupervised clustering and downstream analysis was implemented following the Seurat $\mathrm{R}$ package. Differential expression evaluated based on the non-parametric Wilcoxon rank sum test. Key genes determine by an adjusted $\mathrm{p}$ value of $<0.05$ based on bonferroni correction and $\mid$ avg $\log 2 \mathrm{FC} \mid>0.8$.

Results Preliminary analysis identifies 15 clusters with distinct CD45 immune phenotypes (figure 1). Differential gene expression analysis by hashtag antibody (treatment group) reveals 14 clusters differentially expressing key genes, including 3 clusters upregulated in DIPG compared to NML, and 2 clusters upregulated in irradiated tumors compared to SHAM and NML (figure 2). Notably, analysis demonstrates an individual cluster upregulated in FLASH versus all other groups $(\mathrm{p}=3.07 \mathrm{E}-$ 171). Further deconvolution of specific immune phenotypes represented by each cluster is ongoing.

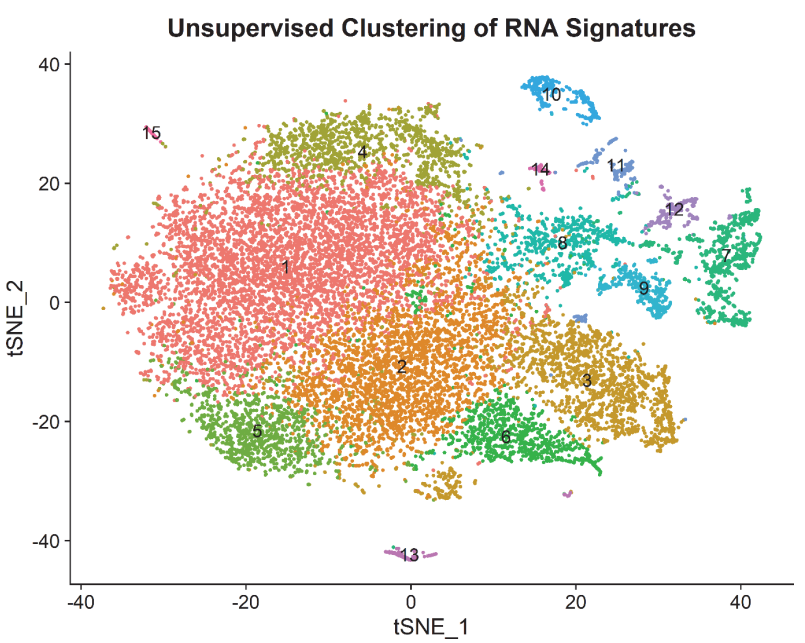

Abstract 91 Figure 1 tSNE plot based on clustering of RNA signatures, grouped by RNA

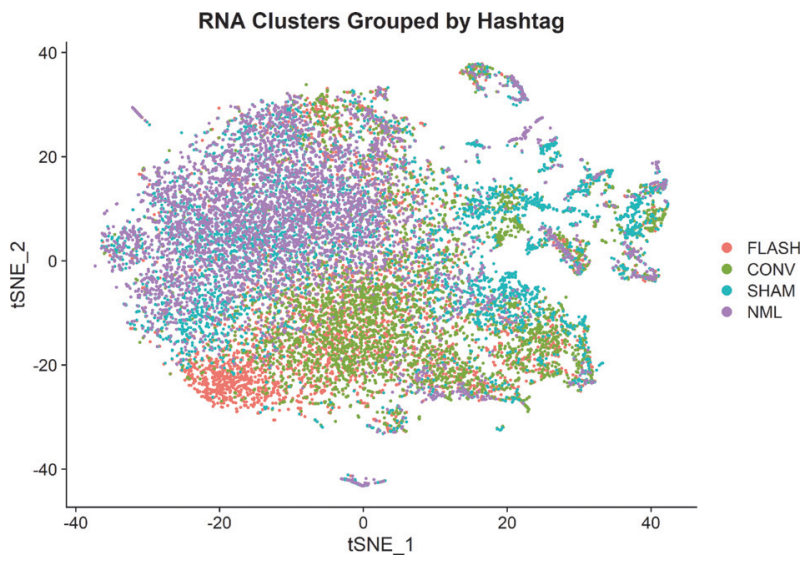

Abstract 91 Figure 2 tSNE plot based on clustering of RNA signatures, grouped by hashtag antibody

Conclusions Our preliminary analysis shows differential immune responses among DIPG tumors compared to NML. We also find several immune cell subsets that are unique to DIPG treated with CONV or FLASH compared to unirradiated samples. Most notably, we identify a single immune cell subset that is exclusive to FLASH alone, indicating that FLASH elicits a unique immune response in murine DIPG.

http://dx.doi.org/10.1136/jitc-2021-SITC2021.091 